\title{
Transcriptional Interference and Gene Orientation in Yeast Noncoding RNA Connections
}

\author{
M. Gullerova AND N.J. Proudfoot \\ Sir William Dunn School of Pathology, University of Oxford, Oxford OX1 3RE, United Kingdom \\ Correspondence: Nicholas.proudfoot@path.ox.ac.uk
}

\begin{abstract}
The compressed nature of genes in yeast requires that transcription units be well defined to prevent read-though transcription from one gene into an adjacent gene. Failure to terminate transcription may result in transcriptional interference of downstream-positioned genes. Transcriptional interference has been studied in several budding yeast gene systems and, interestingly, may be used as a general mechanism of gene regulation. The realization that extensive numbers of noncoding RNAs are transcribed between and across protein-coding genes greatly increases the complexity and subtlety of gene regulation through transcriptional interference. This may be achieved by somehow directly blocking RNA polymerase access to promoters or by the formation of repressive chromatin structures. Furthermore, in fission yeast, read-through transcription from convergent genes may generate double-strand RNAs. These will elicit RNA interference, resulting in heterochromatin formation and consequent gene silencing. Although much remains to be learned from yeast, it is apparent that higher eukaryotes also use related transcriptional interference and gene-silencing strategies. Even though protein-coding genes in mammals are widely separated along chromosomes, extensive numbers of noncoding RNAs are also synthesized. These may well connect distant genes and thus promote transcriptional interference and gene silencing, as is now well established for yeast.
\end{abstract}

The budding yeast Saccharomyces cerevisiae and the fission yeast Schizosaccharomyces pombe have been intensively investigated during the last few decades. As with many other eukaryotes, a milestone in their genetic analysis came with the completion of their genomic sequences. Although many aspects of their gene regulation mechanisms are substantially different, they share general genomic features of unicellular organisms by having energetically efficient, compressed genome organization. That is, they possess very short intergenic regions, usually $<1 \mathrm{~kb}$, and possess few and often short introns (Dujon 1996; Springer et al. 1997; Wood et al. 2002). This gene arrangement contrasts with higher eukaryotes, in which genes are highly intronic and normally separated by large intergenic regions. The very dense arrangement of yeast genomes may exacerbate the possibility of transcriptional interference between these closely spaced genes. In particular, for tandem genes, which are oriented in the same direction, failure to terminate transcription at the end of the first gene will result in inhibition of the next gene. It has been shown that defects in $3^{\prime}$-end formation cause read-through transcription, which leads to transcriptional interference of downstream tandem genes (Shearwin et al. 2005). RNA polymerase II (Pol II) is thought to block access of transcription initiation factors to the promoter region of the downstream gene (Greger et al. 2000). Similarly, for convergent genes, defects in transcription termination may result in collision of elongating Pol II complexes, with consequent inhibition of gene expression (Prescott and Proudfoot 2002). A new feature of gene organization in all eukaryotes is that as well as protein-coding genes, many Pol II transcripts are noncoding (nc) RNAs and may derive from either intergenic, antisense genic, or read-though transcription into genic sequence
(David et al. 2006; Mercer et al. 2009). The presence of these multitudinous ncRNAs raises many new questions regarding the potential for transcriptional interference and the suppression of protein-coding genes by ncRNA transcription. It is apparent that ncRNAs in yeast have major roles in regulating protein-coding gene expression. However, it appears that for most yeast ncRNAs, their function may be to control other genes rather than to encode functional gene products.

\section{TRANSCRIPTION INTERFERENCE BETWEEN GAL GENES IN BUDDING YEAST}

GAL7 and GAL10 genes are part of the GAL gene cluster of $S$. cerevisiae and are required for metabolic conversion of galactose into glucose-6-phosphate, followed by glycolysis (Fridovich-Keil and Jinks-Robertson 1993). This gene pair has been used as a model system to study transcriptional interference (Greger and Proudfoot 1998; Greger et al. 2000; Prescott and Proudfoot 2002). GAL7 and GAL10 genes are arranged in tandem orientation (Fig. 1A). Gal4, a transcriptional activator, has an important regulatory function in expression of $G A L$ genes. It binds as a dimer to the $\mathrm{UAS}_{\mathrm{G}}$ sites in $G A L$ gene promoters and induces transcription (Xu et al. 1995). Gal4 function is prevented by the repressor Gal80, which acts by blocking the activation domain of Gal4 in the absence of galactose (Leuther and Johnston 1992).

The possibility that transcription interference can occur between tandem genes was initially suggested by a study showing that placement of the strong $A C T 1$ promoter (derived from the $A C T 1$ gene) upstream of the $A R O 4$ poly(A) signal caused inhibition of a downstream-positioned HIS7 gene. This effect was enhanced when combined with dele- 
A

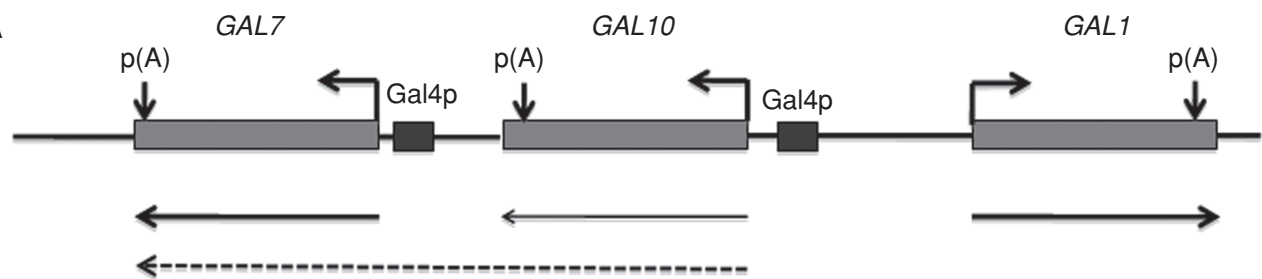

B $\quad \mathrm{pYC10-7}$

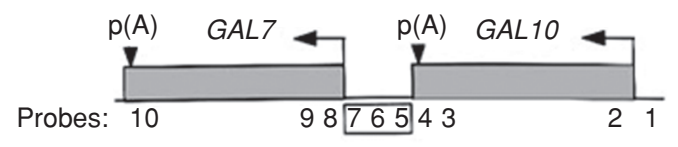

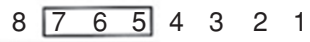

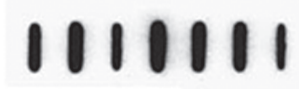

C $\quad \mathrm{p} \triangle T A T A_{\mathrm{G} 7}$

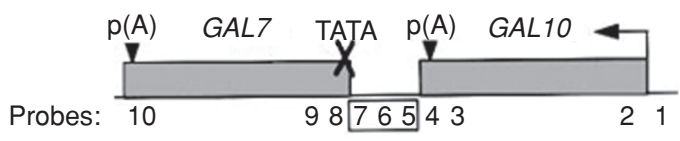

$\begin{array}{llllllll}8 & 7 & 6 & 5 & 4 & 3 & 2 & 1\end{array}$

1111111

D $\mathrm{p} \Delta-\mathrm{UAS}_{\mathrm{G} 10}$

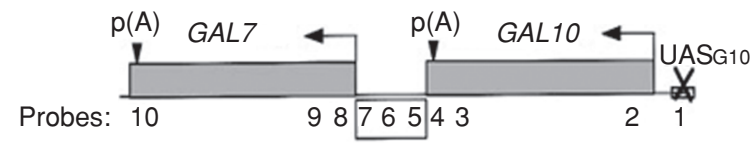

$\begin{array}{lllllll}8 & 7 & 6 & 5 & 4 & 3 & 2\end{array}$

Figure 1. Transcriptional interference between tandem GAL10-7 genes of S. cerevisiae. (A) Diagram of GAL gene cluster in $S$. cerevisiae. The three $G A L$ genes (gray boxes) and their transcriptional orientation (small arrows) are indicated. Long arrows below represent the respective $G A L$ transcripts, with the thickness indicating mRNA levels. The dashed arrow represents the bicistronic $G A L 10-7$ transcript. (Black boxes) Gal4p-binding sites; $[\mathrm{p}(\mathrm{A})]$ the poly $(\mathrm{A})$ sites (black vertical arrows). (B) Transcriptional interference between $G A L 10$ and GAL7 genes. Transcriptional run-on (TRO) analysis of pYC10-7-transformed cells is shown. Positions of single-strand M13 probes are shown below the gene map and above the slots on the hybridization filter. The gene map shows GAL genes as gray boxes with the positions and direction of promoters and $\mathrm{p}(\mathrm{A})$ signals indicated. $(C)$ TRO of $\mathrm{p} \Delta \mathrm{TATA}_{\mathrm{G}}$-transformed cells as in $B$. The boxed numbers $(5-7)$ represent the single-strand DNA (M13) probes spanning the intergenic region. (Black cross) Deletion of the $G A L 7$ TATA-box. (D) TRO on p $\triangle$ $\mathrm{UAS}_{\mathrm{G} 10}$-transformed cells as in $B$. (Black cross) Deletion of the GAL10 UAS G10. (Modified from Greger and Proudfoot 1998.)

tion of the poly(A) site (Springer et al. 1997). GAL10 and GAL7 are highly transcribed genes and therefore provide a good physiological example to study transcription interaction between adjacent genes. TRO analysis revealed active polymerases in the 600-bp intergenic region between GAL10 and GAL7 genes, both on the chromosomal locus as well as on a centromeric plasmid (Fig. 1B). To map the GAL10 terminator region, the GAL7 TATA-box was deleted, completely inactivating GAL7 transcription
(Greger and Proudfoot 1998). Although most polymerases terminate after the GAL10 poly(A) signal, a fraction of elongating polymerases continues transcribing into GAL7 (Fig. 1C). These polymerases produce long read-through transcripts, resulting in bicistronic mRNA (Greger and Proudfoot 1998). More interestingly, the GAL10 termination region is affected by promoter activity. Deletion of GAL10 $\mathrm{UAS}_{\mathrm{G}}$ induces transcription termination directly after the GAL10 poly(A) site, and only a small fraction of 
read-through transcription can be detected (Fig. 1D). Consequently, GAL7 mRNA levels showed more prominent signals than GAL10 at the steady-state level. This may be because the polymerase transcribing the GAL7 promoter region derives from $G A L 10$ transcription as well as from de novo recruitment. The GAL10 gene may also be negatively affected by the adjacent $G A L 1$, which is in a divergent orientation so that these two genes will have adjacent promoters. Consequently, GAL1 may also have a role in the regulation of $G A L 7$ transcription. Overall, these gene arrangements lead to a well-balanced pattern of GAL gene transcription.

To test whether gene orientation is a crucial feature for transcription regulation, the GAL7 and GAL10 genes were changed from tandem to convergent arrangement (Prescott and Proudfoot 2002) on centromeric plasmids. TRO analysis of nascent transcription revealed a similar profile to the wild-type (WT; tandem) situation. GAL10 and $G A L 7$ transcription still terminates at the same position regardless of gene orientation (Fig. 2A-C). The amount of read-through transcription drops to minor levels. Interestingly, GAL7 transcription is elevated in this convergent arrangement. This may be a result of the alleviation of pro- moter occlusion caused by GAL10 read-through transcription in the normal tandem arrangement. However, expression of GAL10 is also higher in this convergent arrangement. In this situation, it is difficult to infer an effect of transcriptional interference in regulating transcription level. Possibly other factors are involved.

\section{TRANSCRIPTIONAL INTERFERENCE AND GENE SILENCING CAUSED BY NCRNA TRANSCRIPTION IN BUDDING YEAST}

More recent discoveries of ncRNAs represent another potential mechanism of transcriptional interference. The prediction of ncRNA existence derives from global studies on widespread transcription of intergenic regions in $S$. cerevisiae (Neil et al. 2009; Xu et al. 2009). Normally, ncRNAs are undetected because of rapid degradation by TRAMP and exosome complexes and are therefore referred to as cryptic unstable transcripts (CUTs) (Vanacova et al. 2005; Wyers et al. 2005; Houseley et al. 2007, 2008). Unexpectedly, antisense ncRNAs were shown to derive from GAL10 coding sequence under repressive conditions (Houseley et al. 2008). This ncRNA extends across the en-

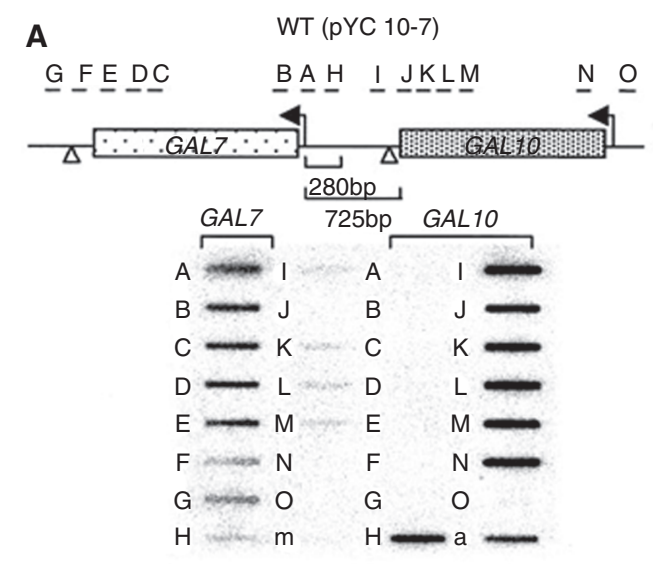

B Con (pYC10-7con)
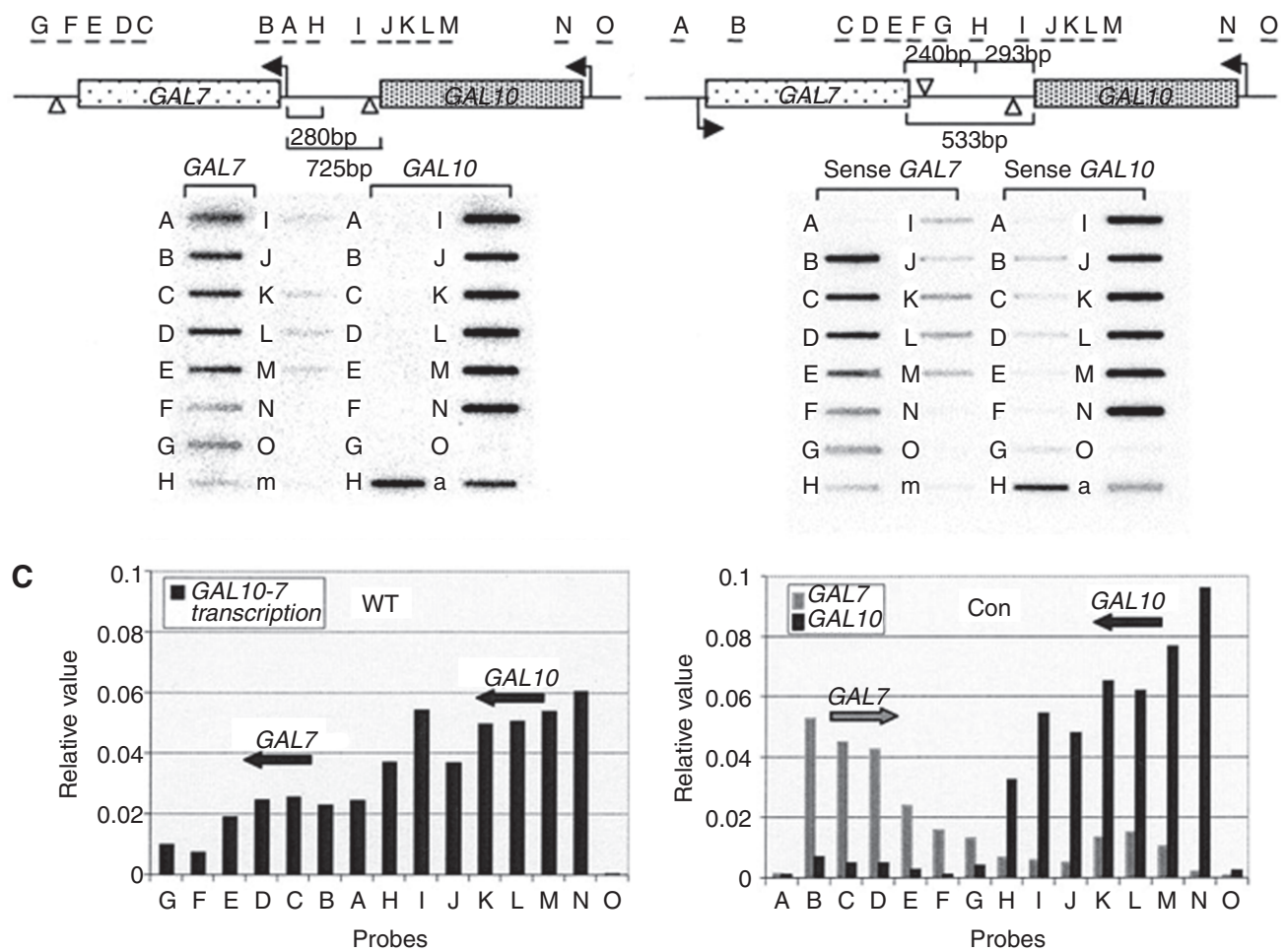

Figure 2. Transcriptional interference between reoriented, convergent GAL genes of S. cerevisiae. (A) TRO analysis of pYC10-7 (WT) transformed cells. The diagram shows the tandem arrangement of GAL10-7, with the direction of transcription indicated by an arrow. Positions of the M13 probes used are indicated. Probe $\mathrm{m}$ is an M13 probe without an insert (negative hybridization control). Probe a is an M13 probe containing ACT1 sequence (positive transcription control). Distances between the ORFs of GAL10 and GAL7 in the WT orientation are indicated. Open arrowheads indicate polyadenylation sites. The GAL7 promoter region is also shown. (B) TRO analysis of pYC10-7Con (Con)-transformed cells. The diagram shows reoriented convergent GAL10 and GAL7, with the intergenic region indicated. This construct includes $240 \mathrm{bp}$ downstream from GAL7 and 293 bp downstream from GAL10 to make a hybrid intergenic region. Sense and antisense M13 DNA probes were selected as appropriate. (C) Quantitation of the data shown in $A$ and $B$. Signals were quantified in a PhosphorImager (Molecular Dynamics). The values were corrected for background hybridization and $U$ content in each probe. Signals were normalized to an $A C T 1$-positive transcription probe (a) on each filter to allow direct comparison of transcription levels. (Modified from Prescott and Proudfoot 2002.) 
tire GAL10 and GAL1 genes with its transcription promoted from regions containing the DNA-binding protein Reb1 (Fig. 3A). Reb1 is an essential protein, initially identified as a Pol I transcription factor (Morrow et al. 1990). Further studies showed that Reb1 also stimulates Pol II transcription of many genes (Remacle and Holmberg 1992; Schuller et al. 1994; Packham et al. 1996). Consensus Reb1-binding sites were detected in many intergenic regions, suggesting that the role of Reb1 in promoting ncRNA transcription is not solely restricted to the $G A L$ cluster (Harbison et al. 2004). Despite the fact that GAL10 ncRNA is transcribed only infrequently (once per hour), it leads to histone modifications, which are normally associated with strongly transcribed regions. In particular, histone H3 K36 trimethylation has been detected over the entire region covered by this antisense ncRNA. H3K36me3 is recognized by Eaf3 protein (Joshi and Struhl 2005), which, in turn, recruits the Rpd3S complex, leading to histone deacetylation across the GAL1-10 locus and consequent repression of the divergent GAL1-10 promoter (Fig. 3A) (Joshi and Struhl 2005; Houseley et al. 2008). Also, $\mathrm{H} 3 \mathrm{~K} 4$ dimethylation and trimethylation (K4me2/3), which are normally associated with active transcription, have been shown to attenuate the GAL1 promoter. Unstable RNAs (referred to as GALluncut in Pinskaya et al. 2009; equivalent to GAL10-1 antisense ncRNA in Housley et al. 2008), initiated upstream of GAL1 in glucose, deposit a repressive $\mathrm{H} 3 \mathrm{~K} 4 \mathrm{me} 2 / 3$ chromatin mark on the GAL1 promoter. This leads to delayed Pol II recruitment and transcription inhibition. GALluncut is dependent on the previously mentioned Reb1. Lack of Reb1 causes a decrease in $\mathrm{H} 3 \mathrm{~K} 4 \mathrm{me} 2 / 3$ in the GAL1 locus, which, in turn, stimulates $G A L 1$ induction. Overall, these data imply that low levels of $G A L$ antisense ncRNA act to set up repressive chromatin structures (Pinskaya et al. 2009).

ncRNA-mediated gene regulation has also been suggested to occur in the SER3 gene in S. cerevisiae (Fig. 3B). $S E R 3$ encodes 3-phosphoglycerate dehydrogenase, which catalyses the first step in serine and glycine biosynthesis. $S E R 3$ is repressed in rich medium. High transcriptional activity in the upstream intergenic regulatory region of the $S E R 3$ gene has been detected under these conditions, resulting in ncRNA production. This ncRNA, called $S R G 1$, interferes with transcription activators across the SER3 promoter region, which thus leads to the transcriptional repression of SER3 (Martens et al. 2004). This process appears analogous to transcriptional interference between tandem genes (e.g., GAL1-10). Furthermore, induction of $S R G 1$ is regulated by serine and requires Cha4, a serinedependent transcriptional activator that binds to the $S R G 1$ promoter. This suggests a physiological role for ncRNA in gene regulation (Martens et al. 2005).

Another example, in which antisense ncRNA has an important regulatory role, is for $I M E 4$, a gene required for entry into meiosis. In haploids, IME4 antisense transcripts are constitutively produced and act to repress IME4 sense transcription and thus block abortive entry into meiosis. In MAT a/ $\alpha$ diploids, IME4 antisense transcription is blocked by the binding of a1/ $\alpha 2$ repressor factors, which, in turn, promote sense transcription from the weaker promoter.
Under appropriate conditions, IME4 sense transcription can be induced to high levels and thus switches yeast from mitosis to meiosis. By this mechanism, dominant antisense transcription in haploids interferes with sense mRNA production in cis. In MAT a/ $\alpha$ diploids, antisense transcription is suppressed by a1/ $\alpha 2$ repressors, resulting in induction of sense transcription (Hongay et al. 2006).

In contrast to the mechanism of transcriptional interference for IME4, a study on $\mathrm{PHO} 4$ proposed stabilization rather than increased levels of antisense transcription (Fig. 4A). In this model, physiological aging of cells is linked to loss of Rrp6 function and consequent stabilization of PHO84 antisense transcripts. This, in turn, leads to repression of sense transcription in cis. Stabilization of PHOS4 antisense RNA is accompanied by recruitment of the histone deacetylase Hda1. This is followed by histone deacetylation and consequent $\mathrm{PHO} 84$ sense transcript repression. In wild-type cells, the exosome component Rrp6 rapidly degrades $\mathrm{PHO} 44$ antisense RNA and thus enhances sense transcription of PHO84. In an Rrp6 deletion mutant or aged wild-type cells, Rrp6 dissociates from PHO84, which, in turn, leads to antisense RNA stabilization. This accumulation correlates with Hda1 recruitment to PHO84 and neighboring loci. However, histone deacetylation mediated by Hdal is restricted to the PHO84 coding region, suggesting a specific gene repression mechanism (Camblong et al. 2007). The generality of this proposed mechanism remains to be clarified by genome-wide analyses of histone $\mathrm{H} 3$ acetylation profiles in the context of Rrp6 occupancy. It is possible that Rrp6 has a key role in gene regulation in response to environmental changes (Camblong et al. 2007).

A study of retrotransposon TY1 CUTs revealed an antisense $T y 1$ CUT called RTL, initiated within the TY1 retrotransposon. It is synthesized by Pol II and degraded by the cytoplasmic $5^{\prime}-3^{\prime}$ exonuclease (Xrn1) decay pathway. Interestingly, $R T L$ represses Ty1 transcription in trans by targeting nascent TY1 RNA. TY1 silencing is partially mediated by histone deacetylation and requires Set1-dependent histone methylation, which resembles heterochromatin gene silencing (Fig. 4C). This was the first described example in S. cerevisiae of RNA-dependent silencing in trans, mediated by chromatin modifications (Berretta et al. 2008).

Although S. cerevisiae lacks an RNA interference (RNAi) pathway (Drinnenberg et al. 2009), transcriptional gene silencing in cis can still occur and is mediated by the accumulation of antisense transcripts. Furthermore, additional experiments on $\mathrm{PHO} 84$ showed that introduction of an additional copy of the PHO84 gene can also lead to silencing of the endogenous allele in trans. This trans repression mechanism is transcriptional and requires trans-acting antisense RNAs. In particular, when $\mathrm{PHO} 4$ is expressed from a plasmid, long antisense RNAs, produced from the 3' end of $\mathrm{PHO} 84$, that extend throughout the promoter region lead to efficient silencing of homologous target loci. In contrast, overproduction of shorter trans-acting antisense RNAs that do not extend into the PHO84 promoter region have only a weak silencing effect (Fig. 4B). A possible explanation for these results may be 
A
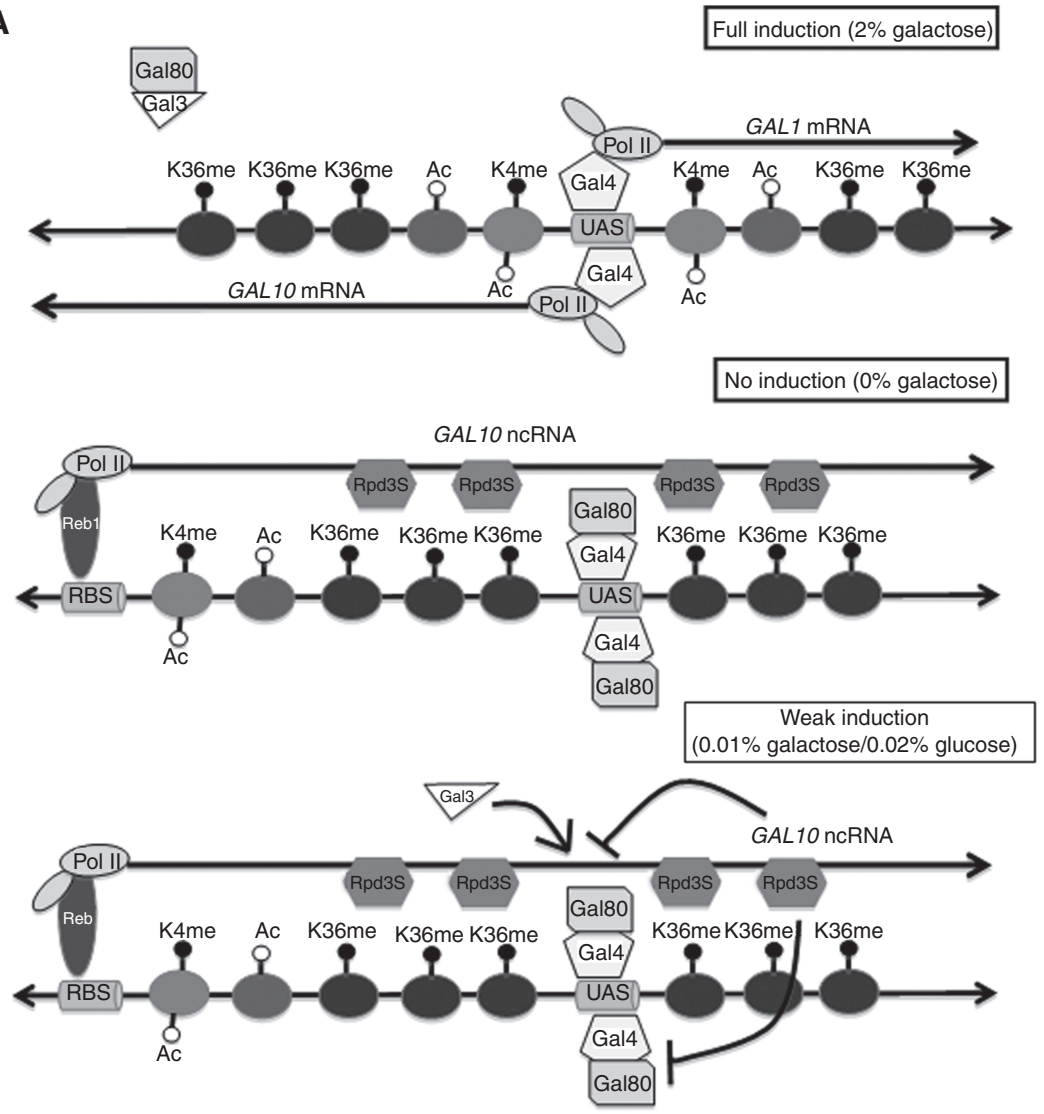

B

High serine levels: biosynthesis off, catabolism on
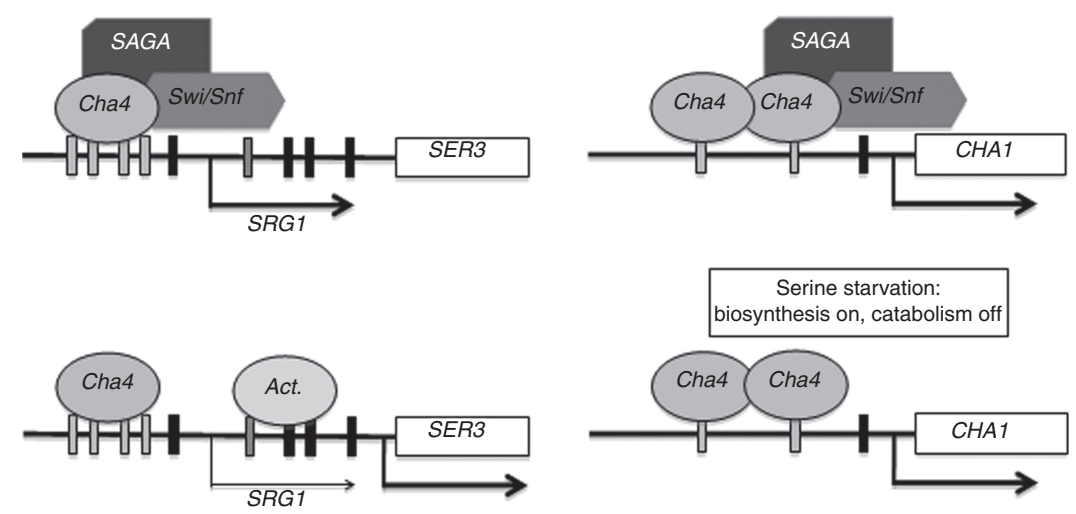

Figure 3. Transcriptional inhibition in S. cerevisiae by noncoding RNAs from the GAL gene cluster and SER3. (A) Model for GAL10 ncRNA-induced histone modifications over the GAL cluster. Full induction: GAL1-10 are transcribed. Because Reb1 binding to the GAL10 3' end is absent, no ncRNA is produced. No induction: GAL1-10 are not transcribed. Reb1 binds within the GAL10 coding region and induces GAL10 ncRNA transcription. This results in high levels of K36me3 and increased histone deacetylation over GAL110. Weak induction: GAL10 ncRNA-induced chromatin modifications repress GAL1-10 transcription under conditions of concomitant weak induction or repression. $(B)$ Model for the coordinated regulation of serine biosynthesis and catabolism by Cha4. In the presence of high serine levels, Cha4 indirectly represses the serine biosynthetic gene SER3 via activation of SRG1 and directly activates the serine catabolic gene CHA1 (upper diagrams). Under serine starvation conditions, when Cha4 is no longer able to recruit SAGA and Swi/Snf, expression states of SER3 and CHAI are reversed (lower diagrams). In this model, expression of SER3 also requires putative activators (Act.) that bind to the previously identified SER3 UAS. In effect, Cha4 can act as both an activator and as a repressor in response to serine. (For GAL gene cluster details, see Houseley et al. 2008; for SER3 details, see Martens et al. 2004.) 
A

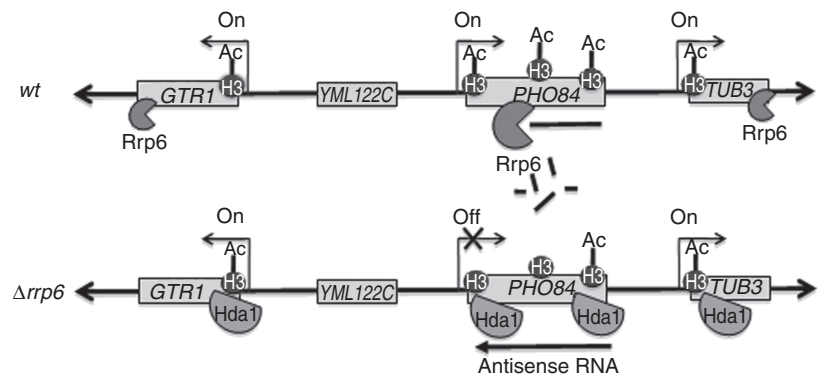

B

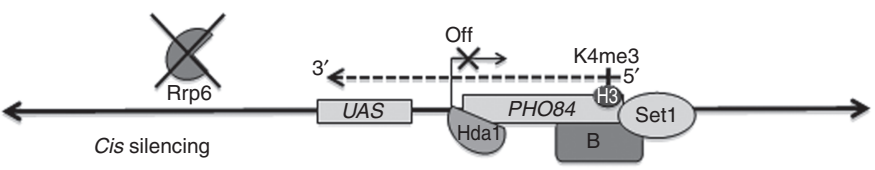

Overexpressed long antisense RNA
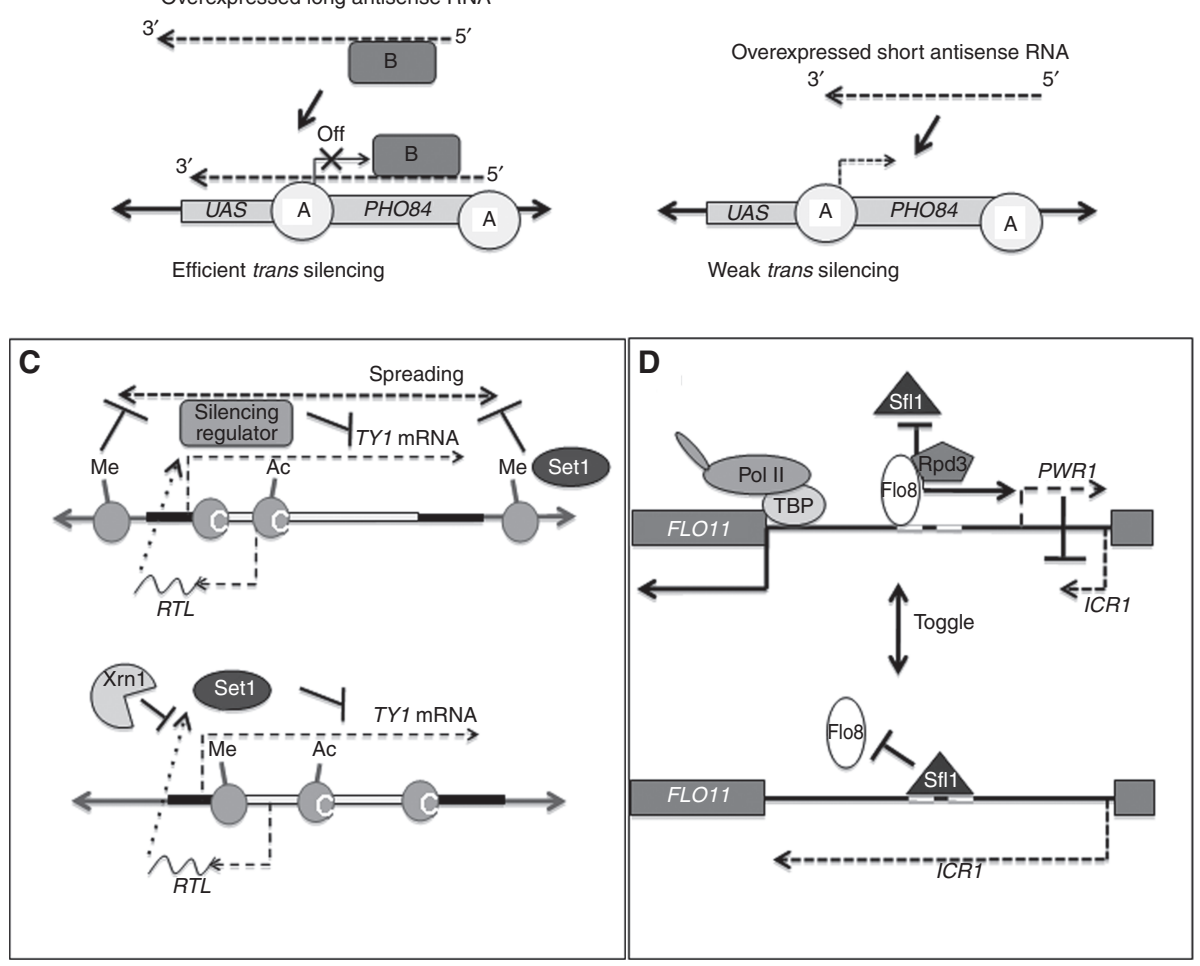

Figure 4. Complex noncoding RNA-mediated transcriptional repression in S. cerevisiae. (A) Model showing that PHO84 antisense RNA stabilization is paralleled by Hdal recruitment, histone deacetylation, and PHO84 transcriptional repression. In wild-type cells, Rrp6 rapidly degrades $\mathrm{PHO} 44$ antisense transcripts, allowing efficient $\mathrm{PHO} 44$ gene transcription (upper diagram). In the absence of Rrp6 or in aging cells (where Rrp6 dissociates from gene), PHO84 antisense transcripts are stabilized (lower diagram). This accumulation correlates with the recruitment of Hda1 to PHO84 and adjacent loci. However, Hda1-induced histone deacetylation is restricted to regions encoding antisense RNA, resulting in specific PHO84 gene repression. (H3-Ac) Acetylated histone H3; (H3) nonacetylated histone H3. (B) Model shows that antisense RNAs can mediate both cis and trans transcriptional gene silencing. Set1 H3K4 methylation promotes antisense RNA production. In cis, antisense RNAs that escape degradation by the nuclear exosome can trigger transcriptional gene silencing in an Hda1/2/3-dependent (PHO84 and VTC3) or Hda1/2/3-independent (GYP5 and YJR129C) manner. In trans, PHO84 antisense RNAs silence the homologous target sequence at the transcriptional level because of a homology region with the UAS promoter sequence. It was proposed that this requires an unidentified silencing factor coating the PHO84 gene (named A). Importantly, although shorter trans-acting antisense RNAs are weak in inducing silencing by A, longer antisense RNAs extending into PHO84 UAS are more effective. It was speculated that a silencing factor (named B) might be recruited by these longer antisense RNAs and would interact with the prebound repressor A enhancing its silencing activity. (C, upper diagram) A model for RNA-dependent Ty1 transcriptional gene silencing indirectly controlled by Set1. If not degraded by the cytoplasmic exonuclease Xrn1, the RTL antisense ncRNA targets an unknown silencing regulator that inhibits $T y l$ transcription through histone deacetylation. Furthermore, the silencing regulator is restricted on $T y 1$ chromatin by Set1-dependent histone methylation, as suggested for heterochromatic domains. In the absence of Set1, silencing factor spreads to adjacent chromatin, allowing Ty1 transcription to be derepressed. (Lower diagram) An alternative model for RNA-dependent Ty1 transcriptional gene silencing directly controlled by Set1. This is largely the same as above, but RTL RNA now activates Set1-dependent histone methylation on Tyl embedded nucleosomes. Histone methylation is then recognized by a putative silencing factor that represses Tyl transcription through histone deacetylation. (Legend continued on facing page.) 
that silencing factors are recruited to the antisense RNA at early stages of transcription and then act to facilitate promoter homology-driven silencing (Camblong et al. 2009). This study suggests the existence of an RNAi-independent mechanism of gene silencing in $S$. cerevisiae.

The discovery of two ncRNAs, ICR 1 and $P W R 1$, which compete with each other in regulation of FLO11 through binding of Sfl1 and Flo8 binding factors (Fig. 4D), suggests that even more complex regulation by ncRNA interference can occur. In this model, long ICR I ncRNA is initiated upstream of FLO11 and is transcribed across the FLO11 promoter, causing repression of FLO11 transcription in cis. This effect is consistent with a promoter occlusion mechanism. Another long PWR 1 ncRNA is initiated on the reverse strand, where it blocks transcription of the interfering ICR I ncRNA and thus promotes FLO11 transcription. This complex mechanism depends on competitive binding of Sfl11 or Flo8 to their cognate DNA-binding sites and their subsequent contribution to either maintain the silent state (binding of Sfl1) or stabilize the active state (binding of Flo8). This mechanism is referred to as "gene toggling." In effect, competition between Sfl1 and Flo8 results in two transcriptional states of the same regulatory region, either active or repressed (Bumgarner et al. 2009).

\section{TRANSCRIPTION INTERFERENCE IN FISSION YEAST}

Transcription termination in eukaryotes normally occurs downstream from the poly(A) site (Proudfoot and Brownlee 1976) and is required to release polymerase from the DNA template into another round of transcription (West et al. 2008). As described above for S. cerevisiae, transcriptional interference arises when elongating polymerases fail to terminate and read into the downstream transcription unit. This may result in reduced expression of the downstream gene through disruption of transcriptional initiation (Irniger et al. 1992; Greger et al. 2000). Transcriptional interference may be particularly deleterious for convergent transcription units. Furthermore, elongating polymerases on both strands could create a potential problem of hybridization between complementary nascent transcripts.

In fission yeast, $S$. pombe, transcription termination has been most studied for ura 4 , which in its chromosomal location is a tandem gene. Three separate elements were defined. Two are site-determining elements, which identify a major poly(A) site and downstream minor poly(A) site. An additional element, located downstream from both poly(A) sites, acts as an enhancer of either poly(A) site. This ele- ment functions in a position-independent and orientationdependent manner but cannot promote termination alone. Furthermore, it can be functionally replaced by the second copy of either site-determining element. The downstream site-determining element and enhancer are defined by specific 16-nucleotide sequences, which are sufficient for ura 4 mRNA 3'-end formation. Elongating polymerases can be detected up to the enhancer sequence but not beyond this point (Humphrey et al. 1994; Birse et al. 1997).

In contrast, TRO analysis of two highly expressed $S$. pombe genes, $n m t 1$ and $n m t 2$, unexpectedly revealed that elongating polymerases transcribe far beyond the poly(A) signal in both cases reading through downstream convergent transcription units (Fig. 5). In contrast to tandem genes, expression of downstream convergent genes avn2 and gut 2 appeared unaffected by read-through transcription. It followed from these results that transcription termination after the poly(A) site may not be strictly required for expression of convergent genes (Hansen et al. 1998).

Analysis of $3^{\prime}$-end formation signals of $n m t 1$ and $n m t 2$ proved that not only the poly(A) site but also downstream transcriptional pause sites were required for efficient termination (Aranda and Proudfoot 1999). For $n m t 2$, the function of the poly(A) signal was shown to be enhanced by pause signals, located at the $3^{\prime}$ end of the convergent avn 2 gene. Deletion of either poly(A) site or pause element increased transcriptional read-through (Fig. 6A). Although $n m t 1$ has a functional poly(A) site, efficient transcriptional termination requires additional pause elements. Insertion of ura 4 RNA 3'-end signals downstream from $n m t 1$ poly(A) site leads to more efficient transcriptional termination (Fig. 6B). The lack of such a pause element in the wild-type $n m t 1$ terminator region was used to explain the extensive levels at nascent read-through transcription observed for this gene (Hansen et al. 1998).

Interestingly, analysis of both ura 4 and $n m t 23^{\prime}$-end formation signals identified downstream elements, with pausing activity, even though they do not share sequence homology. However, both function in the same location and are orientation-specific. The $n m t 2$ pause element is composed of multiple, degenerate sequences that act together to stimulate polymerase pausing. In contrast, the ura 4 pause site is a more compact structure (Aranda and Proudfoot 1999). A model in which transcriptional pause sites stimulate termination is in agreement with the established close connection between polyadenylation and transcription. Pausing polymerases in close proximity to the poly(A) site may facilitate the exchange of transcription factors and potential modifications to the RNA polymerase II elongation complex. Furthermore, pausing will provide more time for exonuclease cleavage of the Pol II-

\footnotetext{
Figure 4. (Continued) $(D)$ Model for transcriptional variegation at the FLO11 locus that involves competition or "toggle" between the ncRNAs ICRI and PWRI. Competitive binding of Sfl1 or Flo8 at their respective binding domains on the DNA initiates events that contribute to either a switch to the silenced FLO11 state (Sfl1 binding) or a switch to the competent state (Flo8 binding). Competition between Sfl1 and Flo8 determines which of two mutually exclusive ncRNA transcription programs occur. ICR 1 represses $F L O 11$ transcription, whereas $P W R 1$ promotes it. Localized chromatin condensation by $R p d 3$ at an upstream site ( 1250 bp) could hinder the access of Sfl1 to its binding site, but promote Flo8 binding, "toggling" the FLO11 promoter toward a state competent for transcription of the protein-coding ORF. (For details, see $A$, Camblong et al. 2007; B, Camblong et al. 2009; $C$, Berretta et al. 2008; $D$, Bumgarner et al. 2009.)
} 

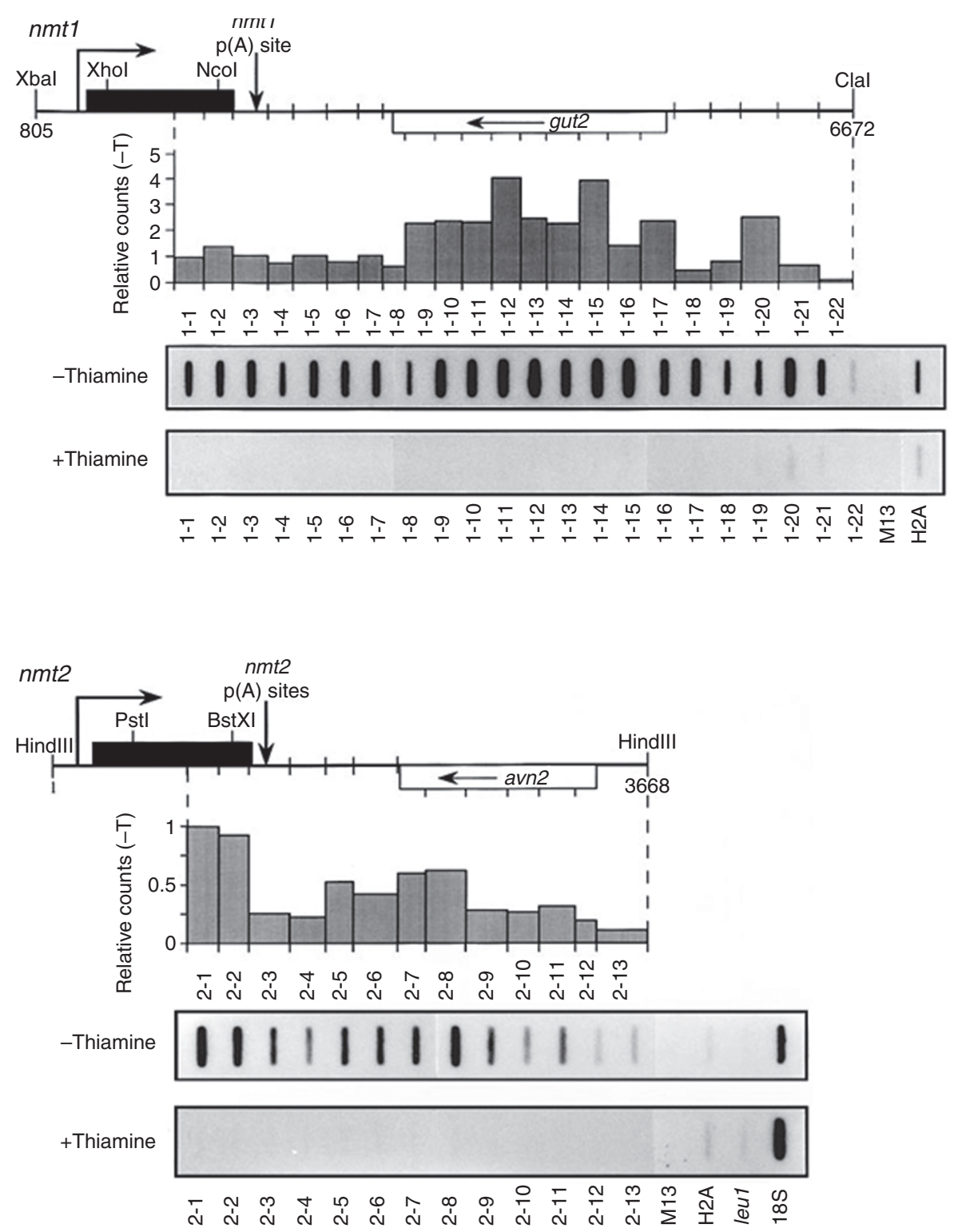

Figure 5. The $n m t 1$ and $n m t 2$ genes of $S$. pombe generate extended transcription into their $3^{\prime}$-flanking sequences. Transcription runon analysis of $n m t 1$ and $n m t 2$ is presented. The wild-type strain $972 \mathrm{~h}-$ was grown overnight in minimal medium in the presence or absence of thiamine, and TRO analysis was performed. Note that thiamine completely represses both $n m t 1$ and $n m t 2$ transcription. The signals obtained after high-stringency wash of the filter are shown and displayed graphically after quantitation. They were corrected for the M13 background (M13mp18 without an insert) and for the U content of the nascent RNA hybridizing to each probe and are expressed relative to probe 1-1. Graph is drawn to scale such that the width of each bar reflects the length of the probe and the height corresponds to the relative average signal across the probe. The H2A gene probe is included as an internal control. (Modified from Hansen et al. 1998.)

associated nascent transcripts following $3^{\prime}$-end processing at the poly(A) signal. Degradation of this transcript up to the Pol II complex is known to promote Pol II termination (West et al. 2008).

The occurrence of transcriptional read-through at convergent genes in $S$. pombe raises many questions. Firstly, why is transcriptional termination inefficient, and what is the generality of this phenomenon? More recently, we have confirmed that several $S$. pombe convergent genes, including $n m t 1$ and $n m t 2$, display transcriptional readthrough (Gullerova and Proudfoot 2008). Convergent gene read-through transcription results in double-strand (ds)
RNA formation, which, in turn, triggers the well-characterized nuclear RNAi response (Moazed 2009). This results in heterochromatin formation especially over centromeric and telomeric regions of the $S$. pombe genome. Interestingly, for convergent genes, this heterochromatin structure is highly transient, only being present in $G_{1}$. This is because these convergent genes only synthesize read-through transcripts in the $G_{1}$ phase of the cell cycle. In $\mathrm{G}_{2}$, the transcriptional profile changes so that elongating polymerase now terminate after the proximal poly(A) site (Fig. 7). Significantly, this transcriptional switch is dependent on the ring-shaped protein complex, 
A

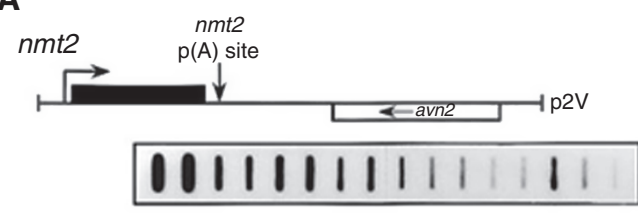

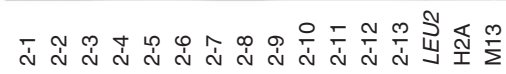

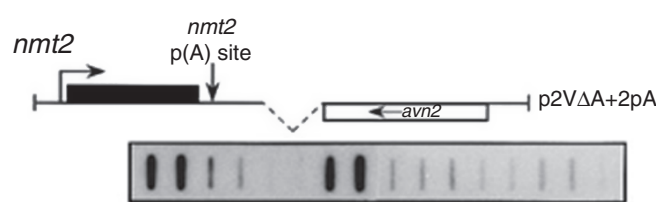

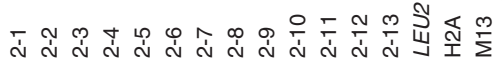

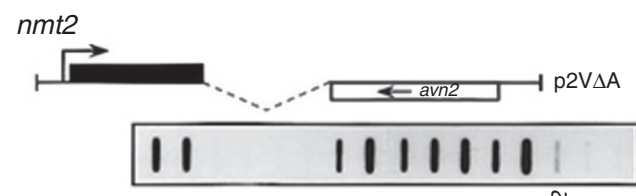

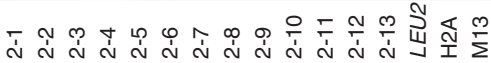

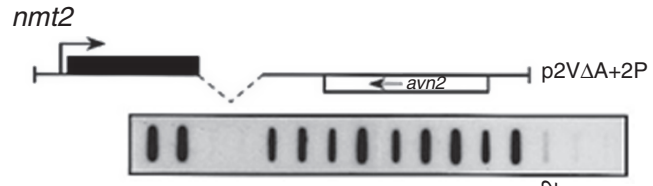

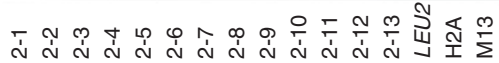

B
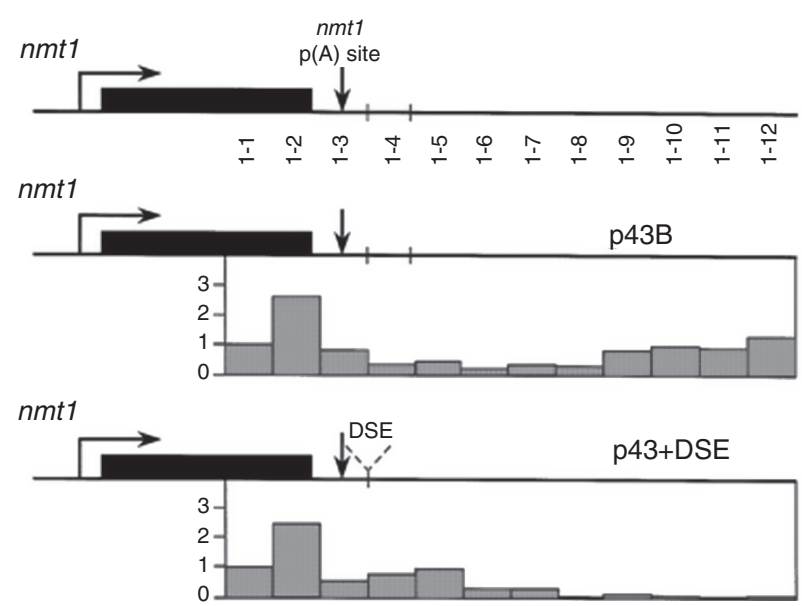

Figure 6. Definition of transcriptional pause sites required for Pol II termination in S. pombe. (A) Manipulation of the $n m t 2$ polymerase profile. Diagrams of each construct used to transform $n m t 2$ disruption strain Sp92 are shown including the location of probes used for TRO analysis. The dotted lines indicate sequences deleted from a particular construct with respect to plasmid p2V. The TRO hybridization signals after an RNase A wash (used to reduce nonspecific background signal) are shown below each gene construct. leu2 and $h 2 a$ probes were included as internal controls (for details, see Hansen et al. 1998). (B) Manipulation of the $n m t 1$ Pol II profile. The constructs indicated were used to transform nmt1 disruption strain Sp204, and TRO analysis was performed. Signals were quantitated (following RNase A treatment) and corrected for M13 background (M13mp18 without an insert) and U content of the nascent RNA hybridizing and expressed relative to the signal for probe $1-1$. The vertical arrow indicates the major $n m t 1$ poly(A) site. Either the ura 4 DSE or ura 4 poly(A) signals were cloned into the PmlI restriction enzyme cleavage site 112 bp downstream from the $n m t 1$ poly(A) site (for details, see Aranda and Proudfoot 1999).

called cohesin (Gullerova and Proudfoot 2008). This multi-subunit protein complex has essential roles in sister chromatid cohesion and faithful chromatid segregation into daughter cells (Hirano 2006). Cohesin is initially loaded onto chromosomes during the $\mathrm{S}$ phase at positions determined by the $\mathrm{Scc} 2 / \mathrm{Scc} 4$ (Mis4/Ss13) cohesin loading complex. Convergent gene $\mathrm{G}_{1}$-specific heterochromatin may also have a role in cohesin recruitment. From these positions it slides into the intergenic regions of convergent genes, as shown in both $S$. cerevisiae and S. pombe. This cohesin relocation is dependent on active transcription, because changes in transcription lead to the repositioning of cohesin (Lengronne et al. 2004; Gullerova and Proudfoot 2008). It is thought that Pol II complexes can't simply pass through the cohesin ring. Consequently, cohesin is pushed from both sides by transcription to a central location between convergent genes. In effect, this accumula- tion of cohesin causes a transcriptional blockade that pauses the polymerase and thus stimulates transcription termination (Fig. 8A). This model complements the mechanism of transcription termination normally stimulated by transcriptional pause sites. Perhaps, in these situations, pause elements are missing. However, the biological function of such a genome design remains to be established.

Histones are crucial components of chromatin. Their modified variants are incorporated into nucleosomes and characterize specialized chromatin. One such conserved histone variant, H2A.Z, has been linked to various cell processes (Guillemette and Gaudreau 2006) and more recently to suppression of antisense transcription in $S$. pombe (Zofall et al. 2009). H2A.Z is recruited to the $5^{\prime}$ ends of genes by the Swr1 complex. Interestingly, deletion of H2A.Z causes accumulation of antisense RNAs, resulting from read-through transcription for some convergent 
A

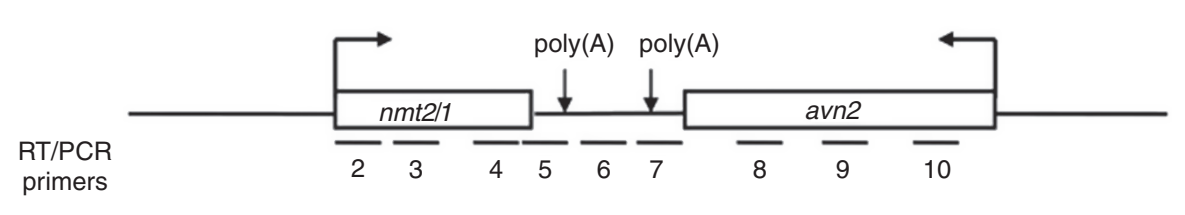

qRT-PCR

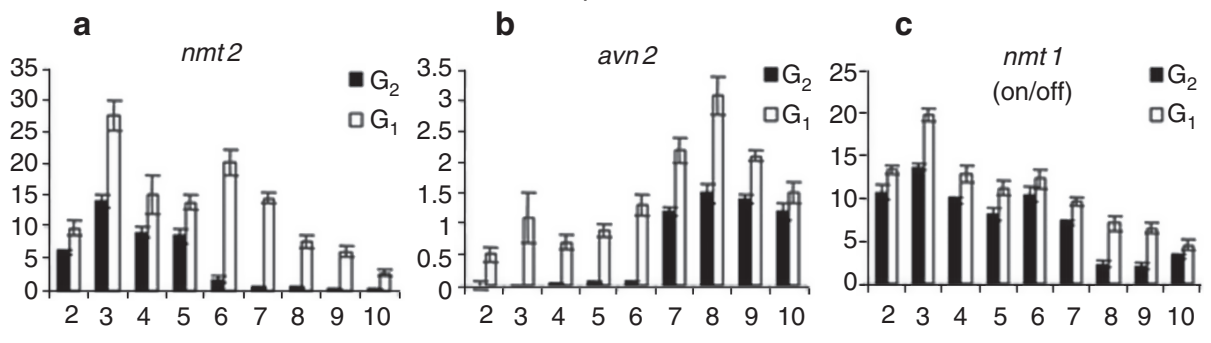

B

3'-RACE
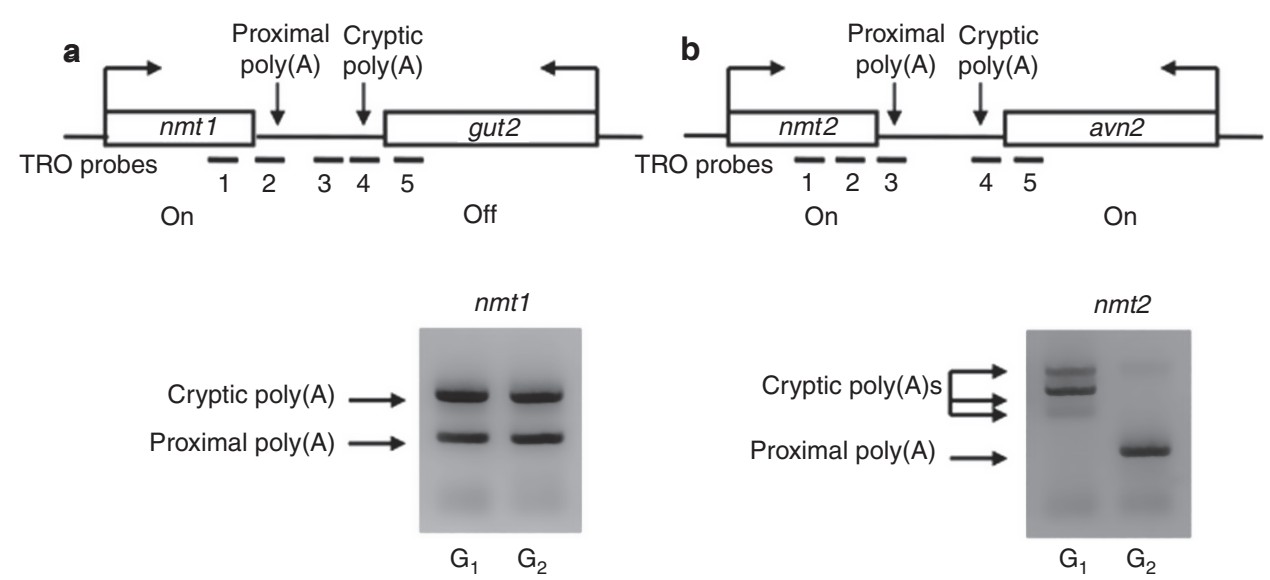

C

Transcription run-on

a

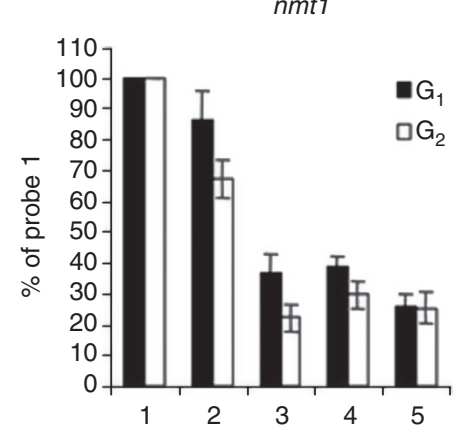

b

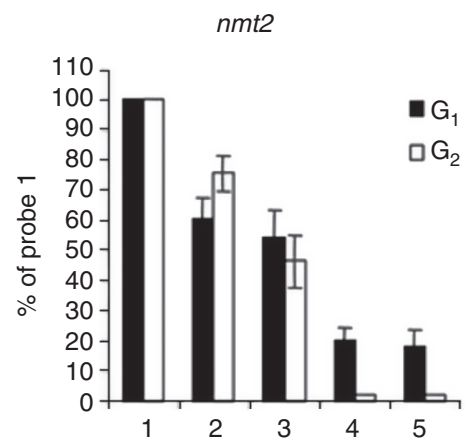

Figure 7. Gene-proximal pA site of $S$. pombe convergent genes is selected in the cell cycle $\mathrm{G}_{2}$ phase. $(A)$ Diagram of primers used for RT-PCR analysis of $n m t 1$-gut 2 and $n m t 2-a v n 2$ transcription. Temperature-sensitive mutants $c d c 10$ and $c d c 25$ were grown in EMM medium and blocked in $G_{1}$ or $G_{2}$ by temperature shift. Total RNA was isolated, and steady-state levels of mRNA were determined by quantitative real-time RT-PCR. Error bars represent \pm SD from triplicate experiments. Graphs $(a-c)$ show mRNA levels for the specified gene. (Black bars) mRNA levels in $\mathrm{G}_{2}$ phase; (white bars) mRNA levels in $\mathrm{G}_{1}$ phase. $(B) \mathrm{G}_{1}$ - or $\mathrm{G}_{2}$-specific mRNAs (as in $A$ ) were analyzed by $3^{\prime}$-RACE: $n m t 1$ gene $(a)$, nmt 2 gene $(b)$. Transcripts using proximal or cryptic pA sites are indicated. (C) Transcription runon analysis of synchronized cells. (a) nmt1 gene; (b) nmt2 gene. Positions of M13 single-stranded probes are shown in Figure 5 (horizontal lines with numbers). In graphs, (black bars) $\mathrm{G}_{1}$ cells; (white bars) $\mathrm{G}_{2}$ cells. TRO signals were normalized to probe 1 and expressed as percent of probe 1. Values in graphs were determined as averages of three independent biological experiments. (Modified from Gullerova and Proudfoot 2008.) 
A
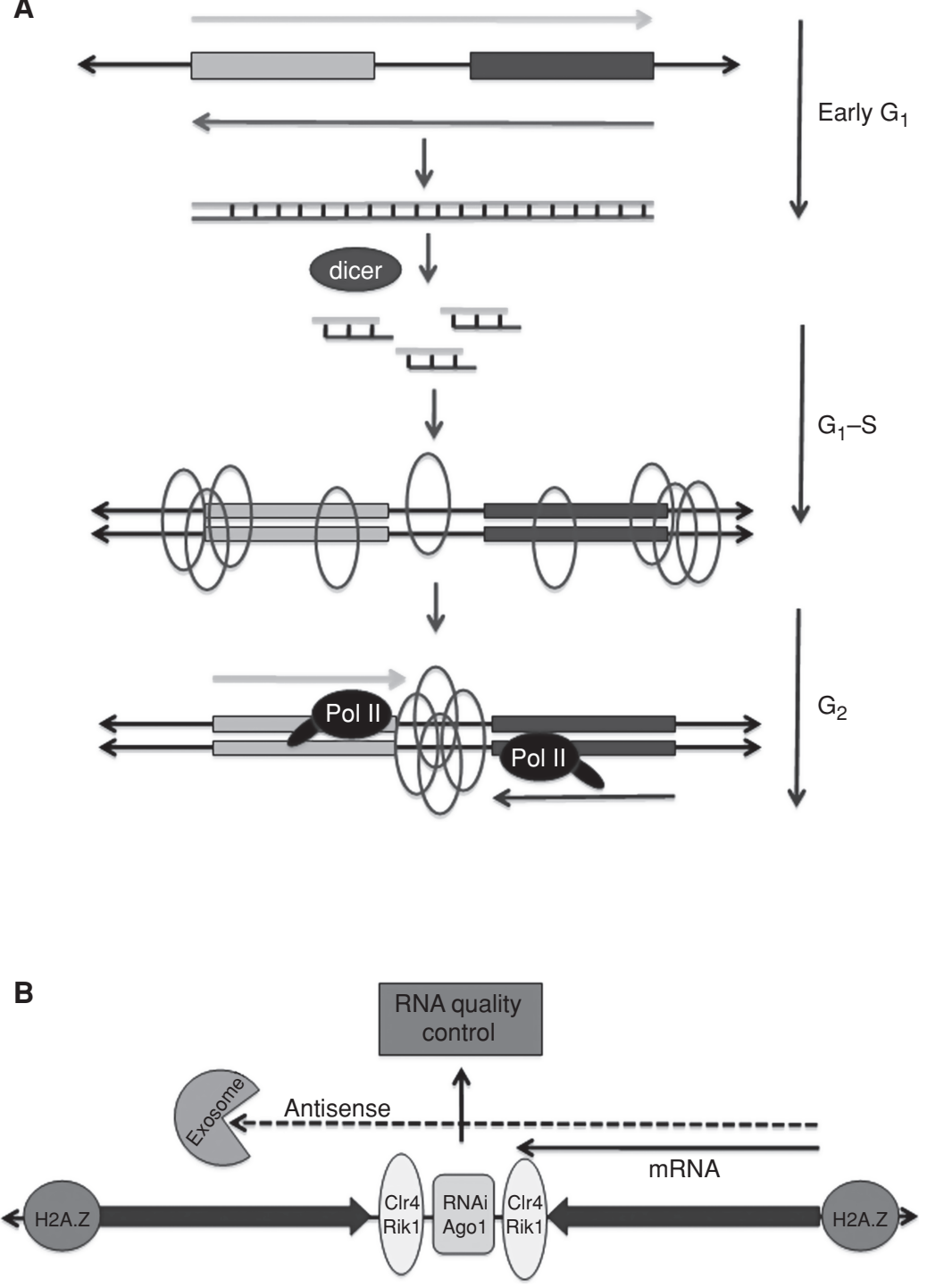

Figure 8. Mechanisms for suppression of convergent gene transcription by noncoding RNAs in S. pombe. (A) Model showing that cohesin accumulation between convergent genes leads to transcription termination in $\mathrm{G}_{2}$. Convergent gene pairs generate bidirectional read-through transcripts in early $\mathrm{G}_{1}$ and consequent dsRNA formation, which is recognized and processed by Dicer into siRNAs. RITS complex is activated and together with histone methyltransferase acts to methylate H3K9 over convergent gene loci. During replication, heterochromatin is removed, allowing $\mathrm{H} 3$ acetylation and thus promoting transcription. Cohesin present between convergent genes in $\mathrm{G}_{2}$ blocks Pol II elongation into the intergenic region, effectively promoting transcriptional termination following the proximal poly(A) site. The relocalized cohesin complex accumulates between convergent genes until the $M$ phase following $G_{2}$, when it is degraded during chromosomal separation (for details, see Gullerova and Proudfoot 2008). (B) Model for antisense suppression at convergent genes. H2A.Z at the $5^{\prime}$ ends of genes contributes to suppression of read-through transcripts that are degraded by the exosome. Antisense suppression also requires $\mathrm{ClrC}$ (Rik1 and Clr4) and Ago1, which together with $\mathrm{H} 2 \mathrm{~A} . \mathrm{Z}$ may facilitate loading of other factors to block Pol II progression and/or mediate the processing of RNAs by the exosome (for details, see Zofall et al. 2009).

genes. H2A.Z was shown to cooperate with RNAi components. Thus, loss of Clr4 methyltransferase or the Argonaute Agol has only a small effect on antisense transcription. However, cells lacking either of these RNAi components together with H2A.Z show a significant increase in ncRNAs levels, which are normally degraded by the exosome (Fig. 8B). Although the precise mechanism remains to be defined, H2A.Z, together with RNAi factors, may have a critical role in degradation of antisense RNAs by the exosome (Zofall et al. 2009; Grewal 2010).
Recently, antisense transcription has been further implicated in primal RNA biogenesis. Bidirectional transcription from DNA repeats produces sense and antisense RNAs, which are then processed by the exosome. These priRNAs target Argonaute to complementary transcripts, which then lead to small interfering (si) RNA amplification and gene silencing. Significantly, priRNA production is Dicer- and RNA-dependent, RNA polymerase-independent. This suggests that the exosome-mediated degradation of antisense transcripts has at a least partial role in 
siRNA production and subsequent gene silencing (Halic and Moazed 2010).

\section{CONCLUSIONS}

The above account charts the data that have so far accumulated on the effect of interactions between transcription units in yeast. Initially, it was shown that protein-coding genes transcribed by Pol II need to rely on effective termination signals to prevent transcription interference or occlusion occurring between adjacent genes. This relatively simple process has now been greatly complicated by the discovery of ncRNAs that have dramatic roles in gene regulation of neighboring genes by transcription interference and/or gene silencing mechanisms. Although ncRNA-mediated gene regulation is clearly an important process in yeast, the situation in higher eukaryotes is only beginning to be appreciated and is likely to be much more complex. The vast stretches of intergenic, repetitive, and intronic sequences that in combination compose most of a higher eukaryotic genome are also now thought to be transcriptionally active (Mercer et al. 2009). Whether these ncRNAs similarly regulate gene expression, as in yeast, is an area of expanding interest and focus. Examples where this is already well known to occur include ncRNAs associated with X-chromosome inactivation and the suppression of paternal or maternal imprinted gene loci (Wan and Bartolomei 2008; Koerner et al. 2009; Lee 2009; Tian et al. 2010). At the same time, ncRNAs are also known to have important roles as functional molecules that act to directly regulate gene expression, such as the ever-expanding class of microRNAs (Bartel 2009). It is clear that studies on transcriptional interference and gene silencing in yeast will provide valuable paradigms for how higher eukaryotes such as humans use this wealth of transcriptional information to regulate gene expression from ever-evolving genomes.

\section{ACKNOWLEDGMENTS}

The authors are supported by a project grant from Cancer Research UK and a Programme grant from the Wellcome Trust. Thanks also to previous laboratory colleagues Ingo Greger, Elizabeth Prescott, Karen Hansen, and Agustin Aranda, whose work is partly republished here.

\section{REFERENCES}

Aranda A, Proudfoot NJ. 1999. Definition of transcriptional pause elements in fission yeast. Mol Cell Biol 19: 1251-1261.

Bartel DP. 2009. MicroRNAs: Target recognition and regulatory functions. Cell 136: 215-233.

Berretta J, Pinskaya M, Morillon A. 2008. A cryptic unstable transcript mediates transcriptional trans-silencing of the Ty1 retrotransposon in S. cerevisiae. Genes Dev 22: 615-626.

Birse CE, Lee BA, Hansen K, Proudfoot NJ. 1997. Transcriptional termination signals for RNA polymerase II in fission yeast. EMBO J 16: 3633-3643.

Bumgarner SL, Dowell RD, Grisafi P, Gifford DK, Fink GR. 2009. Toggle involving cis-interfering noncoding RNAs controls variegated gene expression in yeast. Proc Natl Acad Sci 106: 18321-18326.

Camblong J, Iglesias N, Fickentscher C, Dieppois G, Stutz F. 2007.
Antisense RNA stabilization induces transcriptional gene silencing via histone deacetylation in S. cerevisiae. Cell 131: 706-717.

Camblong J, Beyrouthy N, Guffanti E, Schlaepfer G, Steinmetz LM, Stutz F. 2009. Trans-acting antisense RNAs mediate transcriptional gene cosuppression in S. cerevisiae. Genes Dev 23: 1534-1545.

David L, Huber W, Granovskaia M, Toedling J, Palm CJ, Bofkin L, Jones T, Davis RW, Steinmetz LM. 2006. A high-resolution map of transcription in the yeast genome. Proc Natl Acad Sci 103: 5320-5325.

Drinnenberg IA, Weinberg DE, Xie KT, Mower JP, Wolfe KH, Fink GR, Bartel DP. 2009. RNAi in budding yeast. Science 326: 544 550 .

Dujon B. 1996. The yeast genome project: What did we learn? Trends Genet 12: 263-270.

Fridovich-Keil JL, Jinks-Robertson S. 1993. A yeast expression system for human galactose-1-phosphate uridylyltransferase. Proc Natl Acad Sci 90: 398-402.

Greger IH, Proudfoot NJ. 1998. Poly(A) signals control both transcriptional termination and initiation between the tandem GAL10 and GAL7 genes of Saccharomyces cerevisiae. EMBO J 17: 4771-4779.

Greger IH, Aranda A, Proudfoot N. 2000. Balancing transcriptional interference and initiation on the GAL7 promoter of Saccharomyces cerevisiae. Proc Natl Acad Sci 97: 8415-8420.

Grewal SI. 2010. RNAi-dependent formation of heterochromatin and its diverse functions. Curr Opin Genet Dev 20: 134-141.

Guillemette B, Gaudreau L. 2006. Reuniting the contrasting functions of H2A.Z. Biochem Cell Biol 84: 528-535.

Gullerova M, Proudfoot NJ. 2008. Cohesin complex promotes transcriptional termination between convergent genes in $S$. pombe. Cell 132: 983-995.

Halic M, Moazed D. 2010. Dicer-independent primal RNAs trigger RNAi and heterochromatin formation. Cell 140: 504-516.

Hansen K, Birse CE, Proudfoot NJ. 1998. Nascent transcription from the $n m t 1$ and $n m t 2$ genes of Schizosaccharomyces pombe overlaps neighbouring genes. EMBO J 17: 3066-3077.

Harbison CT, Gordon DB, Lee TI, Rinaldi NJ, Macisaac KD, Danford TW, Hannett NM, Tagne JB, Reynolds DB, Yoo J, et al. 2004. Transcriptional regulatory code of a eukaryotic genome. Nature 431: 99-104.

Hirano T. 2006. At the heart of the chromosome: SMC proteins in action. Nat Rev Mol Cell Biol 7: 311-322.

Hongay CF, Grisafi PL, Galitski T, Fink GR. 2006. Antisense transcription controls cell fate in Saccharomyces cerevisiae. Cell 127: 735-745.

Houseley J, Kotovic K, El Hage A, Tollervey D. 2007. Trf4 targets ncRNAs from telomeric and rDNA spacer regions and functions in rDNA copy number control. EMBO J 26: 4996-5006.

Houseley J, Rubbi L, Grunstein M, Tollervey D, Vogelauer M. 2008. A ncRNA modulates histone modification and mRNA induction in the yeast GAL gene cluster. Mol Cell 32: 685-695.

Humphrey T, Birse CE, Proudfoot NJ. 1994. RNA 3' end signals of the $S$. pombe ura 4 gene comprise a site determining and efficiency element. EMBO J 13: 2441-2451.

Irniger S, Egli CM, Kuenzler M, Braus GH. 1992. The yeast actin intron contains a cryptic promoter that can be switched on by preventing transcriptional interference. Nucleic Acids Res 20: 4733-4739.

Joshi AA, Struhl K. 2005. Eaf3 chromodomain interaction with methylated H3-K36 links histone deacetylation to Pol II elongation. Mol Cell 20: 971-978.

Koerner MV, Pauler FM, Huang R, Barlow DP. 2009. The function of non-coding RNAs in genomic imprinting. Development 136: 1771-1783.

Lee JT. 2009. Lessons from X-chromosome inactivation: Long ncRNA as guides and tethers to the epigenome. Genes Dev 23: 1831-1842.

Lengronne A, Katou Y, Mori S, Yokobayashi S, Kelly GP, Itoh T, Watanabe Y, Shirahige K, Uhlmann F. 2004. Cohesin relocation from sites of chromosomal loading to places of convergent transcription. Nature 430: 573-578.

Leuther KK, Johnston SA. 1992. Nondissociation of GAL4 and 
GAL80 in vivo after galactose induction. Science 256: 13331335.

Martens JA, Laprade L, Winston F. 2004. Intergenic transcription is required to repress the Saccharomyces cerevisiae SER3 gene. Nature 429: 571-574.

Martens JA, Wu PY, Winston F. 2005. Regulation of an intergenic transcript controls adjacent gene transcription in Saccharomyces cerevisiae. Genes Dev 19: 2695-2704.

Mercer TR, Dinger ME, Mattick JS. 2009. Long non-coding RNAs: Insights into functions. Nat Rev Genet 10: 155-159.

Moazed D. 2009. Small RNAs in transcriptional gene silencing and genome defence. Nature 457: 413-420.

Morrow BE, Ju Q, Warner JR. 1990. Purification and characterization of the yeast rDNA binding protein REB1. J Biol Chem 265: 20778-20783.

Neil H, Malabat C, d'Aubenton-Carafa Y, Xu Z, Steinmetz LM, Jacquier A. 2009. Widespread bidirectional promoters are the major source of cryptic transcripts in yeast. Nature 457: 10381042.

Packham EA, Graham IR, Chambers A. 1996. The multifunctional transcription factors Abflp, Rap1p and Reb1p are required for full transcriptional activation of the chromosomal PGK gene in Saccharomyces cerevisiae. Mol Gen Genet 250: 348-356.

Pinskaya M, Gourvennec S, Morillon A. 2009. H3 lysine 4 di- and tri-methylation deposited by cryptic transcription attenuates promoter activation. EMBO J 28: 1697-1707.

Prescott EM, Proudfoot NJ. 2002. Transcriptional collision between convergent genes in budding yeast. Proc Natl Acad Sci 99: 8796-8801.

Proudfoot NJ, Brownlee GG. 1976. 3' Non-coding region sequences in eukaryotic messenger RNA. Nature 263: 211-214.

Remacle JE, Holmberg S. 1992. A REB1-binding site is required for GCN4-independent ILV1 basal level transcription and can be functionally replaced by an ABF1-binding site. Mol Cell Biol 12: 5516-5526.

Schuller HJ, Schutz A, Knab S, Hoffmann B, Schweizer E. 1994. Importance of general regulatory factors Rap1p, Abflp and Reb1p for the activation of yeast fatty acid synthase genes FAS1 and FAS2. Eur J Biochem 225: 213-222.

Shearwin KE, Callen BP, Egan JB. 2005. Transcriptional interference: A crash course. Trends Genet 21: 339-345.

Springer C, Valerius O, Strittmatter A, Braus GH. 1997. The adjacent yeast genes ARO4 and HIS7 carry no intergenic region. $J$ Biol Chem 272: 26318-26324.

Tian D, Sun S, Lee JT. 2010. The long noncoding RNA, Jpx, is a molecular switch for X chromosome inactivation. Cell 143: 390-403.

Vanacova S, Wolf J, Martin G, Blank D, Dettwiler S, Friedlein A, Langen H, Keith G, Keller W. 2005. A new yeast poly(A) polymerase complex involved in RNA quality control. PLoS Biol 3: e189. doi: 10.1371/journal.pbio.0030189.

Wan LB, Bartolomei MS. 2008. Regulation of imprinting in clusters: Noncoding RNAs versus insulators. Adv Genet 61: 207-223.

West S, Proudfoot NJ, Dye MJ. 2008. Molecular dissection of mammalian RNA polymerase II transcriptional termination. Mol Cell 29: 600-610.

Wood V, Gwilliam R, Rajandream MA, Lyne M, Lyne R, Stewart A, Sgouros J, Peat N, Hayles J, Baker S, et al. 2002. The genome sequence of Schizosaccharomyces pombe. Nature 415: 871880 .

Wyers F, Rougemaille M, Badis G, Rousselle JC, Dufour ME, Boulay J, Regnault B, Devaux F, Namane A, Seraphin B, et al. 2005. Cryptic Pol II transcripts are degraded by a nuclear quality control pathway involving a new poly(A) polymerase. Cell 121: $725-737$.

Xu HE, Kodadek T, Johnston SA. 1995. A single GAL4 dimer can maximally activate transcription under physiological conditions. Proc Natl Acad Sci 92: 7677-7680.

Xu Z, Wei W, Gagneur J, Perocchi F, Clauder-Munster S, Camblong J, Guffanti E, Stutz F, Huber W, Steinmetz LM. 2009. Bidirectional promoters generate pervasive transcription in yeast. Nature 457: 1033-1037.

Zofall M, Fischer T, Zhang K, Zhou M, Cui B, Veenstra TD, Grewal SI. 2009. Histone H2A.Z cooperates with RNAi and heterochromatin factors to suppress antisense RNAs. Nature 461: 419-422. 


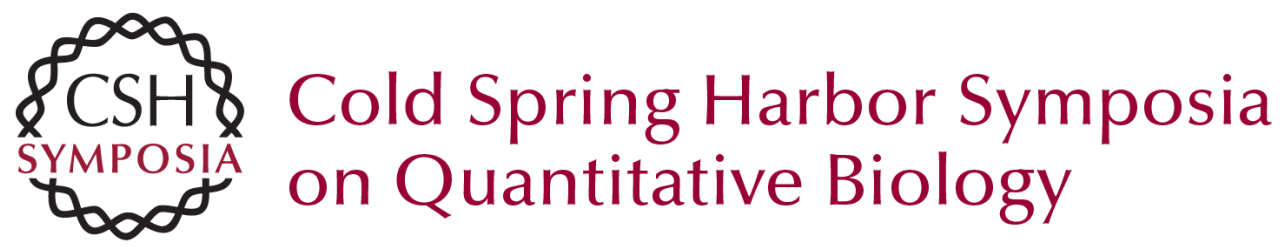

\section{Transcriptional Interference and Gene Orientation in Yeast: Noncoding RNA Connections}

M. Gullerova and N.J. Proudfoot

Cold Spring Harb Symp Quant Biol 2010 75: 299-311 originally published online April 5, 2011

Access the most recent version at doi:10.1101/sqb.2010.75.048

References This article cites 53 articles, 20 of which can be accessed free at:

http://symposium.cshlp.org/content/75/299.full.html\#ref-list-1

\section{License}

Email Alerting Receive free email alerts when new articles cite this article - sign up in the box at the Service top right corner of the article or click here. 